\title{
An Online Tool to Inform and Educate Caregivers on Amyotrophic Lateral Sclerosis (ALS)
}

\author{
Maria GIANNAKI ${ }^{\mathrm{a}, 1}$, Parisis GALLOS ${ }^{\mathrm{a}}$, Joseph LIASKOS ${ }^{\mathrm{a}}$, \\ Spyros ZOGAS ${ }^{\mathrm{a}}$ and John MANTAS ${ }^{\mathrm{a}}$ \\ ${ }^{a}$ Health Informatics Laboratory, School of Health Sciences, National and Kapodistrian \\ University of Athens, Greece
}

\begin{abstract}
Amyotrophic Lateral Sclerosis (ALS) is the most common Motor Neuron Disease. This paper presents the design, development, and evaluation of an online tool that provides information and training materials to caregivers about ALS, to promote health care and quality of life of patients. To collect the appropriate content, a literature review was conducted, and a Content Management System (CMS) was used for the development of the tool. For its evaluation, reliability, acceptance, effectiveness and usefulness were examined through semistructured interviews. The online tool was positively evaluated by the caregivers, who participated in the evaluation process.
\end{abstract}

Keywords. Amyotrophic Lateral Sclerosis, Palliative Care, online systems, education, caregivers

\section{Introduction}

Amyotrophic Lateral Sclerosis (ALS) is the most common Motor Neuron Disease (MND) and a progressive neurodegenerative disease characterized by a rapid progressive paralysis and death within 1-5 years after the onset of symptoms [1,2]. No treatment is available to date. Therefore, the provision of Palliative Care (PC) in patients with ALS, in properly organized structures and services are of major importance for the supportive care of these patients, their families and caregivers [3]. The purpose of this paper is to present the development and the evaluation of an online tool which informs caregivers about ALS and PC, and trains them for caring and improving quality of life of ALS patients.

\section{Methods}

In order to form the content of the online tool, a literature review about ALS and PC was conducted and multimedia content suitable for the caregivers was collected. The design of the tool was chosen after consulting IT professionals and potential users, in

\footnotetext{
${ }^{1}$ Corresponding Author, Maria Giannaki, MSc, Health Informatics Laboratory, School of Health Sciences, National and Kapodistrian University of Athens, Greece; E-mail: giannaki.maro@gmail.com.
} 
order that caregivers have access to the content easily. WordPress, was selected for the tool development, as it provides functionalities including ones needed for this online tool. Regarding the evaluation, qualitative data were collected concerning the reliability, acceptance, effectiveness and usefulness of the tool. Semi-structured interviews were used because they provided participants with some guidance on what to talk about and allowed discovery or elaboration of information that was important to them [4].

\section{Results and Discussion}

A prototype online tool was created in Greek language. Caregivers can access and navigate to its content, by using a common web browser. The content includes text enriched with images, figures, videos, and links to related websites, about ALS, PC and skills that caregivers need to have, to provide improved healthcare services to ALS patients. A contact form is available to the caregivers to communicate with experts in ALS and PC. Six caregivers participated in the evaluation process. They reported that the tool is simple to navigate, easy to access and easy to use. They pointed out the rich, comprehensible, reliable, useful informational and educational content provided. The majority of them agreed that caregivers would consider to use this tool as an additional source of information and training for improving their knowledge and skills in ALS and $\mathrm{PC}$.

\section{Conclusions}

An online tool was developed to provide information about ALS and PC, and training materials to caregivers in order to improve their knowledge, skills, and quality of care. The positive evaluation of this tool reveals the necessity and value of such solutions in raising the educational level of caregivers and subsequently improving the patients' care. A limitation of this study is that due to COVID-19 pandemic the number of users evaluated the tool is quite low, since in person interviews could not be easily performed. Future work includes the upgrade of this tool with additional functions, content, and multilanguage support.

\section{Acknowledgements}

The authors would like to thank the PC Unit "Galilee" for its support on this research.

\section{References}

[1] Cleveland DW, Rothstein JD. From Charcot to Lou Gehrig: deciphering selective motor neuron death in ALS. Nature reviews Neuroscience 2001;2(11):806-819.

[2] Everett EA, Pedowitz E, Maiser S, et al. Top Ten Tips Palliative Care Clinicians Should Know About Amyotrophic Lateral Sclerosis. Journal of Palliative Medicine 2020;23(6):842-847.

[3] Riva N, Agosta F, Lunetta C, Filippi M, Quattrini A. Recent advances in amyotrophic lateral sclerosis. J Neurol. 2016 Jun;263(6):1241-54.

[4] Gill P, Stewart K, Treasure E, Chadwick B. Methods of data collection in qualitative research: interviews and focus groups. Br Dent J. 2008 Mar 22;204(6):291-5. 